Supporting Information

for

\title{
Dual-Mode Photonic Sensor Array for Detecting and Discriminating Hydrazine and
}

\author{
Aliphatic Amines \\ Taihong Liu*, Lüjie Yang, Wan Feng, Ke Liu, Qian Ran, Weina Wang, Quan Liu, Haonan Peng, \\ Liping Ding, and Yu Fang* \\ Key Laboratory of Applied Surface and Colloid Chemistry of Ministry of \\ Education, School of Chemistry and Chemical Engineering, Shaanxi Normal \\ University, Xi'an 710062, People's Republic of China
}

Corresponding authors: liuth121@snnu.edu.cn (T. Liu); yfang@snnu.edu.cn (Y. Fang)

\section{Contents}

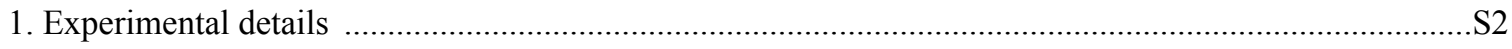

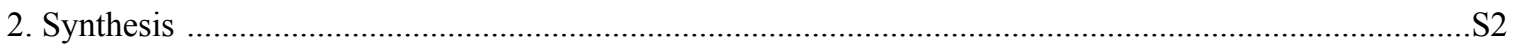

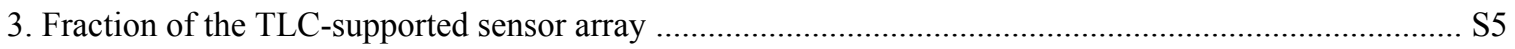

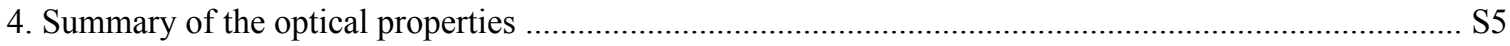

5. Optical properties of NA-3T-CN in different solvents ..................................................................... S5

6. Optimized geometries of the oligothiophene derivatives using DFT calculations …………………........ S6

7. Frontier molecular orbital diagrams of optimized oligothiophene derivatives ……............................... S6

8. Relationship between the UV optical changes of NA-3T-CN and hydrazine concentration in THF ...... S7

9. Sensing performance of NA-3T-CN to methylamine in THF ……...................................................... S7

10. Sensing performance of 3T-CN and 4T-CN to hydrazine in THF …………..................................... S8

11. Optical response of the three spots-based sensor arrays................................................................... S8

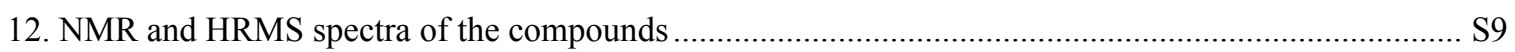

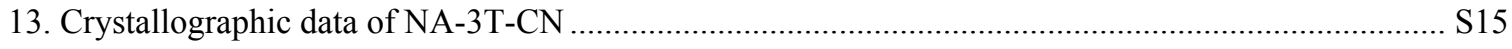

14. A comparison of DL to other reported chemosensors ..................................................................... S16

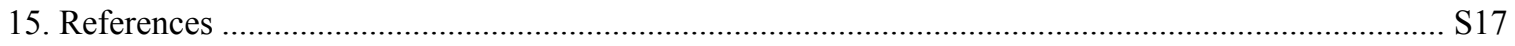




\section{Experimental Section}

All reagent chemicals and solvents were used as purchased without further purification unless otherwise noted. Thin-layer chromatography (TLC) was performed on pre-coated silica gel 60 F254 plates. Column chromatography was performed over silica gel 230-400 mesh. The ${ }^{1} \mathrm{H}$ and ${ }^{13} \mathrm{C}$ NMR measurements were performed at room temperature by a Bruker Avance III NMR spectrometer with tetramethylsilane (TMS) as an internal reference. Fluorescence measurements were performed using a time-correlated single photon counting fluorescence spectrometer (Edinburgh Instruments FLS980). The absolute luminescent quantum yields were estimated by the same spectrometer using an integrating sphere. The high-resolution mass spectra (HRMS) were acquired using a Bruker maXis UHR-TOF mass spectrometer. All the absorption spectra were measured on a Shimadzu UV-2600 spectrometer. Optical band gaps $\left(E \mathrm{~g}_{(\mathrm{Opt.})}\right)$ were determined from $\lambda_{\text {onset. }}$ The solutions were prepared with ultrapure water obtained from a Millipore Milli-Q system.

Single crystal of NA-3T-CN was obtained in THF/n-hexane solution. A suitable crystal was selected and collected on a 'Bruker APEX-II CCD/D8' diffractometer using $\mathrm{Cu}(\mathrm{Ga}$, Mo) $\mathrm{K} \alpha$ radiation. The crystal was kept at $153.0 \mathrm{~K}$ during data collection and the data were corrected for absorption through Gaussian integration from indexing of the crystal faces. Using Olex2, the structure was solved with the XT structure solution program using Intrinsic Phasing and refined with the XL refinement package using Least Squares minimisation. The crystallographic information of NA-3T-CN has been deposited to Cambridge Crystallographic Data Centre and signed as CCDC code 1974256.

\section{Synthesis}

As presented in Scheme S1, the synthesis of dicyanovinyl end-capped oligothiophene derivatives (3T-CN, 4T-CN and NA-3T-CN) were performed through Knoevenagel condensation of aldehydes with malononitrile in the presence of triethylamine. ${ }^{1-3}$ The starting materials $3 \mathrm{~T}-\mathrm{CHO},{ }^{4} 4 \mathrm{~T}-\mathrm{CHO},{ }^{5}$ and NA-3T-CHO,${ }^{6}$ have been synthesized by our group based on the Vilsmeier reaction with phosphorus oxychloride and $N, N$ dimethylformamide (DMF) that allowed us to obtain in a single reaction step. The synthesis methodology of $3 \mathrm{~T}-\mathrm{CN}^{7}$ and $4 \mathrm{~T}-\mathrm{CN}^{8}$ has been described elsewhere. 
General synthetic procedure: Under $\mathrm{N}_{2}$ atmosphere, to a stirred solution of the corresponding formyl-substituted oligothiophene derivatives in EtOH was added an excess amount of malononitrile and several drops of triethylamine as catalyst. After being stirred at $80{ }^{\circ} \mathrm{C}$ for $5 \mathrm{~h}$, the resulting mixture was cooled to room temperature and concentrated under reduced pressure to an appropriate volume. Then the product was collected by centrifugation, washed thoroughly with ethanol, and dried under vacuum to afford dicyanovinyl end-capped target oligothiophene derivatives as dark red solids.

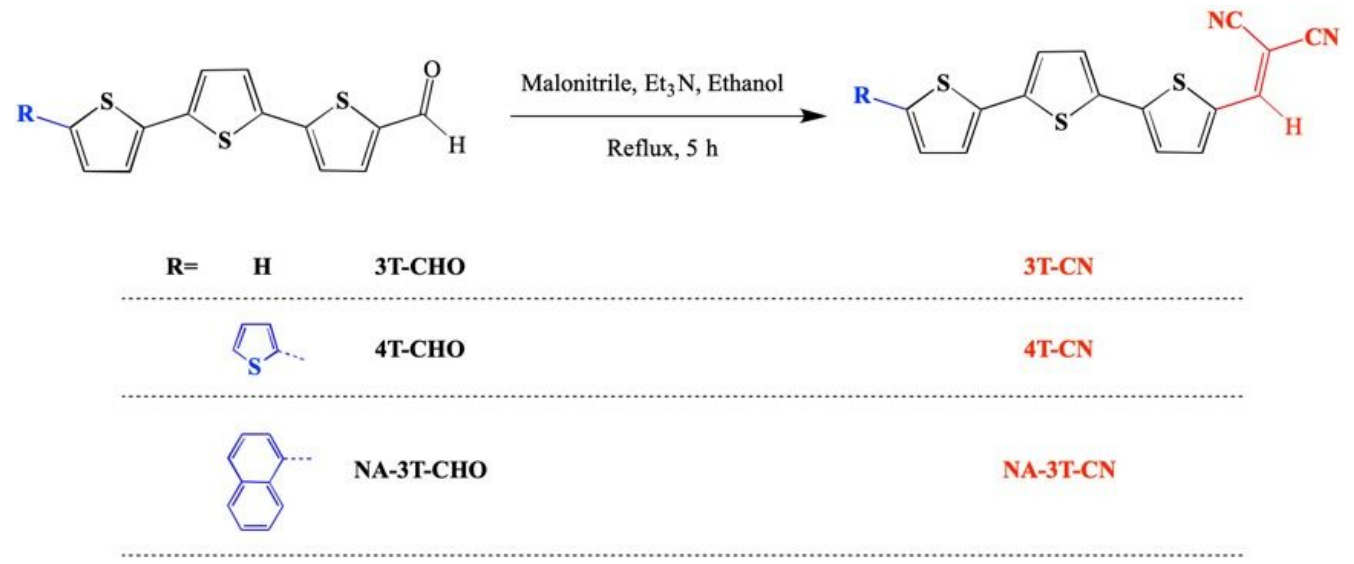

Scheme S1. Synthesis route for the oligothiophene derivatives.

3T-CN: $R_{\mathrm{f}} \sim 0.50(\mathrm{DCM} / n$-hexane, $2 / 1), 82 \%$ yield. ${ }^{1} \mathrm{H}$ NMR $\left(600 \mathrm{MHz}, d_{8}\right.$-THF) $\delta$ : $8.11\left(\mathrm{~s}, 1 \mathrm{H}, \mathrm{CH}=(\mathrm{CN})_{2}\right), 7.65(\mathrm{~d}, J=4.0 \mathrm{~Hz}, 1 \mathrm{H}), 7.40(\mathrm{~d}, J=3.8 \mathrm{~Hz}, 1 \mathrm{H}), 7.32$ (t, $J=$ $4.0 \mathrm{~Hz}, 2 \mathrm{H}), 7.23$ (d, $J=3.2 \mathrm{~Hz}, 1 \mathrm{H}), 7.16$ (d, $J=3.8 \mathrm{~Hz}, 1 \mathrm{H}), 7.00-6.90(\mathrm{~m}, 1 \mathrm{H}) .{ }^{13} \mathrm{C}$ NMR (151 MHz, $d_{8}$-THF) $\delta: 150.70,147.82,140.56,140.08,136.08,133.97,133.65$, 128.12, 128.07, 125.89, 124.93, 124.80, 124.52, 114.10, 113.44, 75.94. HRMS (APCI, $m / z):[\mathrm{M}+\mathrm{H}]^{+}$calcd. for $\mathrm{C}_{16} \mathrm{H}_{9} \mathrm{~N}_{2} \mathrm{~S}_{3}{ }^{+}: 324.9922$; found: 324.9908 .

4T-CN: $R_{\mathrm{f}} \sim 0.47(\mathrm{DCM} / n$-hexane, $2 / 1), 87 \%$ yield. ${ }^{1} \mathrm{H}$ NMR $\left(600 \mathrm{MHz}, d_{8}\right.$-THF) $\delta$ : $9.09\left(\mathrm{~s}, 1 \mathrm{H}, \mathrm{CH}=(\mathrm{CN})_{2}\right), 6.50(\mathrm{~s}, 1 \mathrm{H}), 6.05(\mathrm{~d}, J=4.1 \mathrm{~Hz}, 1 \mathrm{H}), 5.81(\mathrm{~d}, J=3.9 \mathrm{~Hz}, 1 \mathrm{H})$, $5.72(\mathrm{~d}, J=4.1 \mathrm{~Hz}, 1 \mathrm{H}), 5.65(\mathrm{~d}, J=5.1 \mathrm{~Hz}, 1 \mathrm{H}), 5.59 \sim 5.52(\mathrm{~m}, 2 \mathrm{H}), 5.47(\mathrm{~d}, J=3.8 \mathrm{~Hz}$, $1 \mathrm{H}), 5.32(\mathrm{dd}, J=5.0,3.6 \mathrm{~Hz}, 1 \mathrm{H}) .{ }^{13} \mathrm{C} \mathrm{NMR}\left(151 \mathrm{MHz}, d_{8}\right.$-THF) $\delta: 150.65,140.53$, $134.66,134.05,128.24,127.99,127.89,125.61,125.15,125.00,124.58,124.52,124.08$, 76.18. HRMS (APCI, $m / z$ ): $[\mathrm{M}+\mathrm{H}]^{+}$calcd. for $\mathrm{C}_{20} \mathrm{H}_{11} \mathrm{~N}_{2} \mathrm{~S}_{4}{ }^{+}$: 406.9800 ; found: 406.9807.

NA-3T-CN: $R_{\mathrm{f}} \sim 0.90(\mathrm{DCM}), 77 \%$ yield. ${ }^{1} \mathrm{H}$ NMR $\left(600 \mathrm{MHz}, d_{8}\right.$-THF) $\delta: 8.18$ (dd, $J=$ 6.0, 3.6 Hz, 1H), $8.14(\mathrm{~s}, 1 \mathrm{H}), 7.87-7.76(\mathrm{~m}, 2 \mathrm{H}), 7.68(\mathrm{~d}, J=4.1 \mathrm{~Hz}, 1 \mathrm{H}), 7.50(\mathrm{~d}, J=$ 
$7.0 \mathrm{~Hz}, 1 \mathrm{H}), 7.46$ (d, $J=3.9 \mathrm{~Hz}, 1 \mathrm{H}), 7.44-7.39$ (m, 3H), 7.36 (dd, $J=7.7,3.9 \mathrm{~Hz}, 2 \mathrm{H})$, $7.25(\mathrm{~d}, J=3.9 \mathrm{~Hz}, 1 \mathrm{H}), 7.16(\mathrm{~d}, J=3.7 \mathrm{~Hz}, 1 \mathrm{H}) .{ }^{13} \mathrm{C}$ NMR $\left(151 \mathrm{MHz}, d_{8}-\mathrm{THF}\right) \delta$ : $150.68,147.78,142.20,140.55,139.83,136.40,134.21,134.03,133.81,131.51,128.78$, $128.61,128.37,128.24,127.87,126.52,126.02,125.19,125.15,125.13,125.06,124.61$, 114.09, 113.44, 75.99. HRMS (APCI, $m / z$ ): $[\mathrm{M}+\mathrm{H}]^{+}$calcd. for $\mathrm{C}_{26} \mathrm{H}_{15} \mathrm{~N}_{2} \mathrm{~S}_{3}{ }^{+}: 451.0392$; found: 451.0395.

Zn-TPP: As illuminated in Scheme S2, Zn-TPP was obtained in two steps. In the first step, starting material 5,10,15,20-tetrakis(4-hydroxyphenyl)porphyrin (TPP-OH) was converted to TPP in the presence of hexyl bromide and $\mathrm{K}_{2} \mathrm{CO}_{3}$. The purple solid product was obtained by column chromatography with the eluent of DCM and methanol (20:1, v:v). Yield is $\sim 80 \%$. ${ }^{1} \mathrm{H} \mathrm{NMR}\left(\mathrm{CDCl}_{3}, 400 \mathrm{MHz}, \delta, \mathrm{ppm}\right) 8.86(8 \mathrm{H}), 8.10-8.07(8 \mathrm{H})$, 7.25-7.22 (8 H), 4.22-4.11 (8 H), 1.97-1.92 (8 H), 1.63-1.59 (8 H), 1.49-1.41 (16 H), 1.00-0.96 (12 H). ${ }^{13} \mathrm{C}$ NMR ( $\left.\mathrm{CDCl}_{3}, 100 \mathrm{MHz}, \mathrm{ppm}\right) \delta: 158.97,135.61,134.47,119.84$, $112.71,68.33,31.76,29.50,25.95,22.73,14.14$. For the second step, compound TPP was treated with $\mathrm{Zn}(\mathrm{OAc})_{2} \cdot 2 \mathrm{H}_{2} \mathrm{O}$ in the mixture of methanol and dichloromethane $(1: 1, \mathrm{v}: \mathrm{v})$ at refluxing for $3 \mathrm{~h}$. Then the solvent was removed in vacuum. The crude product was checked by TLC with the eluent of dichloromethane and hexane. The pure product was obtained by column chromatography on silica (yield 85\%). ${ }^{1} \mathrm{H}$ NMR $\left(\mathrm{CDCl}_{3}, 600 \mathrm{MHz}\right.$, $\delta$, ppm) $8.97(8 \mathrm{H}), 8.09(8 \mathrm{H}), 7.24(8 \mathrm{H}), 4.33-4.13(8 \mathrm{H}), 2.09-1.85(8 \mathrm{H}), 1.72-1.57$ (8 $\mathrm{H}), 1.51-1.37(16 \mathrm{H}), 1.03-0.93(12 \mathrm{H}) .{ }^{13} \mathrm{C} \mathrm{NMR}\left(\mathrm{CDCl}_{3}, 125 \mathrm{MHz}, \mathrm{ppm}\right) \delta:$ 158.81, 150.54, 135.41, 135.07, 131.91, 120.85, 112.59, 68.33, 31.77, 29.73, 29.52, 25.95, 22.74, 14.15. HRMS (ESI, $m / z$ ): $[\mathrm{M}+\mathrm{H}]^{+}$calcd. for $\mathrm{C}_{68} \mathrm{H}_{77} \mathrm{~N}_{4} \mathrm{O}_{4} \mathrm{Zn}^{+}$: 1077.5231; found: 1077.5226 .

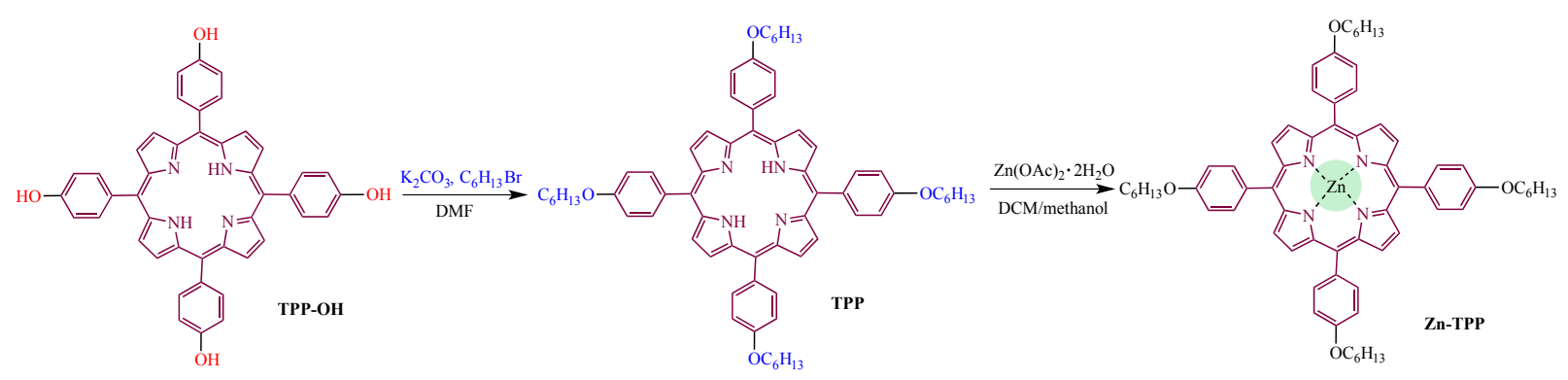

Scheme S2. Synthesis route for the zinc porphyrin derivative (Zn-TPP). 


\section{Fraction of the TLC-supported sensor array}

TLC plates were adopted as the porous and inert substrate to support the fluorophores. Firstly, NA-3T-CN and Zn-TPP in THF $(\mathrm{C} \sim 5.0 \mu \mathrm{M})$ were prepared respectively in a dust-free container for film preparation. And then, a quantitative amount $(6 \mu \mathrm{L})$ of the solution as prepared was added onto the plate surface at room temperature. After spotting the two fluorophores and their mixture in an array format, the film is allowed to dry in the air. Then, certain amount of hydrazine solution with increasing concentrations as described in the figures were then dropped onto the spots and dried in the fume hood for a while before observation and photo-taking. Effect of other solutions was conducted in the same way.

\section{Summary of the optical properties of the oligothiophene derivatives}

Table S1. Main photophysical parameters of the related oligothiophene derivatives.

\begin{tabular}{cccccccccc}
\hline Comps & $\lambda_{\text {abs }}[\mathbf{n m}]$ & $\lambda_{\text {em }}[\mathbf{n m}]$ & $\boldsymbol{\Delta} \lambda[\mathbf{n m}]$ & Solvents & Comps & $\lambda_{\text {abs }}[\mathbf{n m}]$ & $\lambda_{\mathbf{e m}}[\mathbf{n m}]$ & $\Delta \lambda[\mathbf{n m}]$ & Solvents \\
\hline 3T & 357 & 433 & 76 & DCM & NA-3T & 377 & $461 / 481$ & 104 & DCM \\
\hline 3T-CHO & 397 & 499 & 102 & DCM & NA-3T-CHO & 419 & 532 & 113 & DCM \\
3T-CN & 467 & 584 & 117 & THF & NA-3T-CN & 479 & 626 & 147 & THF \\
\hline 4T & 396 & $454 / 482$ & 86 & $\mathrm{CHCl}_{3}$ & $\mathbf{4 T - C N}$ & 487 & 648 & 161 & THF \\
\hline 4T-CHO & 425 & 539 & 114 & $\mathrm{CHCl}_{3}$ & & & & & \\
\hline
\end{tabular}

\section{Optical properties of NA-3T-CN in different solvents}

Table S2. Main photophysical parameters of NA-3T in different solvents.

\begin{tabular}{ccccccc}
\hline Solvents & $\boldsymbol{\Delta f}$ & $\boldsymbol{\varepsilon}_{\max }\left[\mathbf{M}^{-1} \cdot \mathbf{c m}^{-1}\right]$ & $\lambda_{\mathrm{abs}}[\mathbf{n m}]$ & $\boldsymbol{\lambda}_{\mathrm{em}}[\mathbf{n m}]$ & $\Delta \lambda[\mathbf{n m}]$ & $\Delta \boldsymbol{v}\left[\mathbf{c m}^{-1}\right]$ \\
\hline Toluene & 0.0135 & $4.52 \times 10^{4}$ & 484 & 582 & 98 & 3479 \\
\hline Benzene & 0.002 & $5.04 \times 10^{4}$ & 484 & 586 & 102 & 3596 \\
$\mathbf{D C M}$ & 0.217 & $4.21 \times 10^{4}$ & 491 & 640 & 149 & 4741 \\
$\mathbf{T H F}$ & 0.209 & $5.52 \times 10^{4}$ & 480 & 626 & 146 & 4858 \\
$\mathbf{C H C l}_{3}$ & 0.152 & $5.15 \times 10^{4}$ & 495 & 626 & 131 & 4227 \\
\hline Acetone & 0.287 & $5.15 \times 10^{4}$ & 475 & 654 & 179 & 5762 \\
\hline Methanol & 0.309 & $4.92 \times 10^{4}$ & 476 & 665 & 189 & 5970 \\
\hline
\end{tabular}


Figure S1. Lippert plots for NA-3T-CN in kinds of organic solvents.

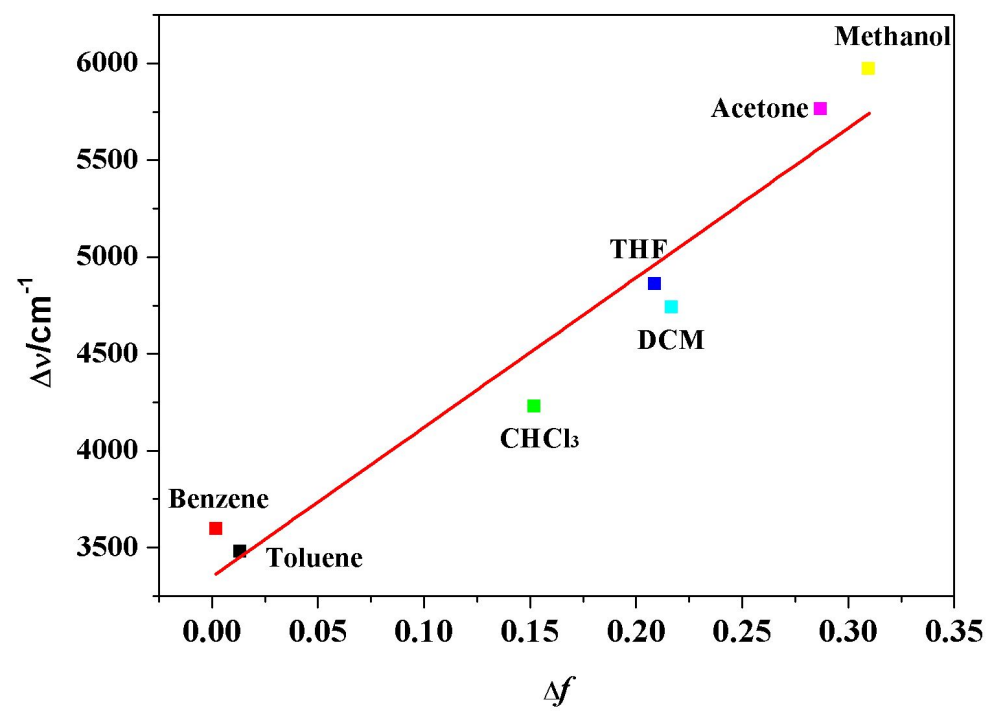

6. Optimized geometries of the oligothiophene derivatives using DFT calculations.

\begin{tabular}{|c|c|c|c|}
\hline & Top View & Side View \\
\hline 3T-CN & TT-CN & &
\end{tabular}

7. Frontier molecular orbital diagrams of optimized oligothiophene derivatives using DFT calculations.

\begin{tabular}{|c|c|c|}
\hline 3T-CN & HOMO & LUMO \\
\hline 4T-CN & 2 & 0 \\
\hline
\end{tabular}


8. Relationship between the UV optical changes of NA-3T-CN and hydrazine concentration in THF

Figure S2. Absorption changes of the NA-3T-CN in THF upon addition of hydrazine.

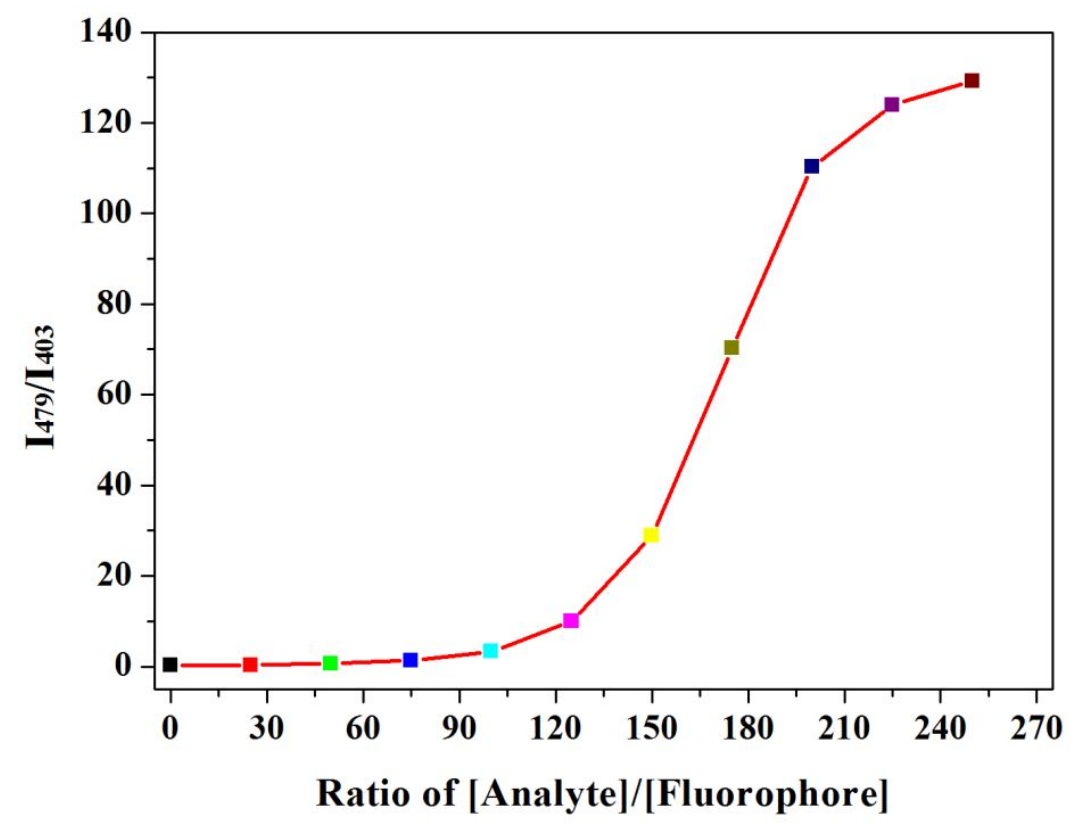

\section{Sensing performance of NA-3T-CN to methylamine in THF}

Figure S3. Absorption changes of the NA-3T-CN $(20.0 \mu \mathrm{M})$ in THF upon addition of methylamine $(0 \sim 60.0 \mathrm{mM})$.

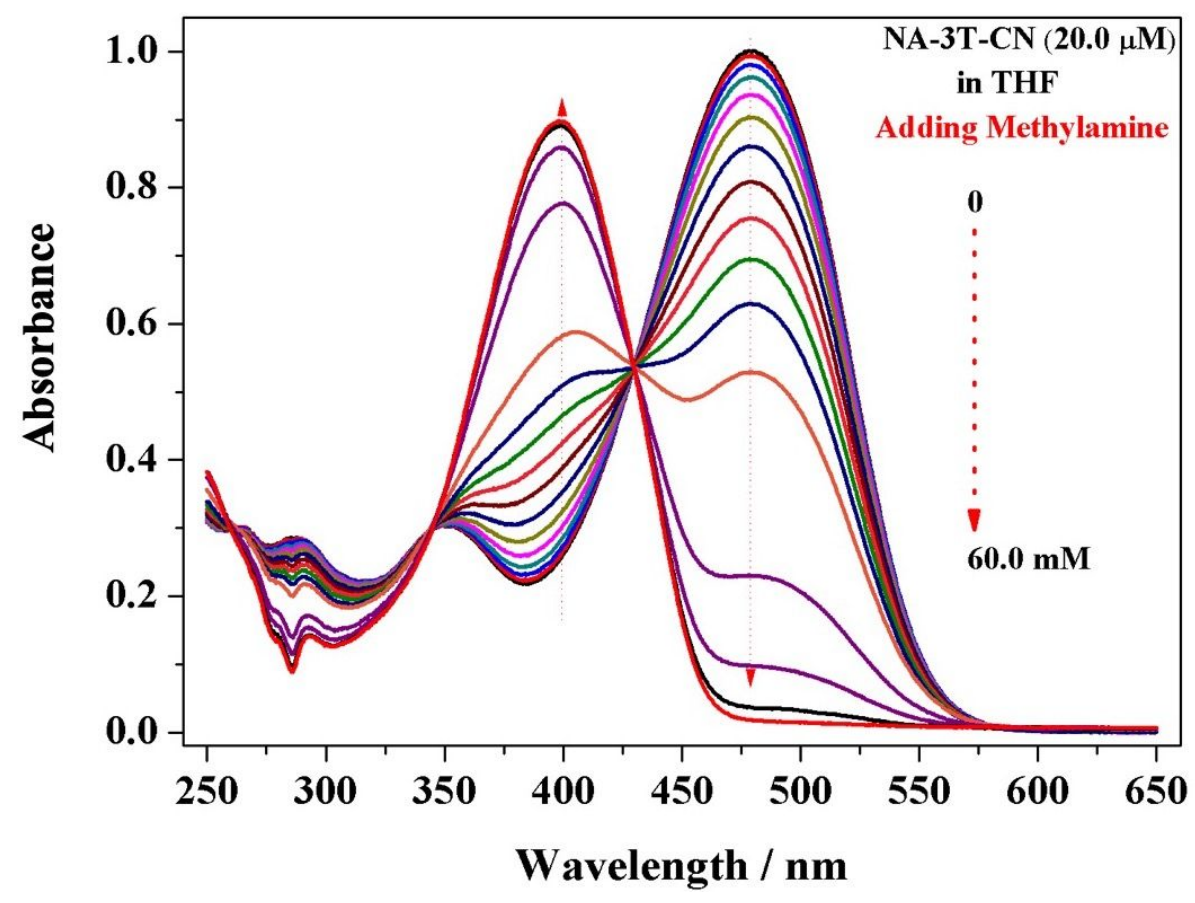




\section{Sensing performance of 3T-CN and 4T-CN to hydrazine in THF}

Figure S4. Absorption changes of the 3T-CN and 4T-CN in THF $(20.0 \mu \mathrm{M})$ upon addition of hydrazine $(0 \sim 60.0 \mathrm{mM})$.
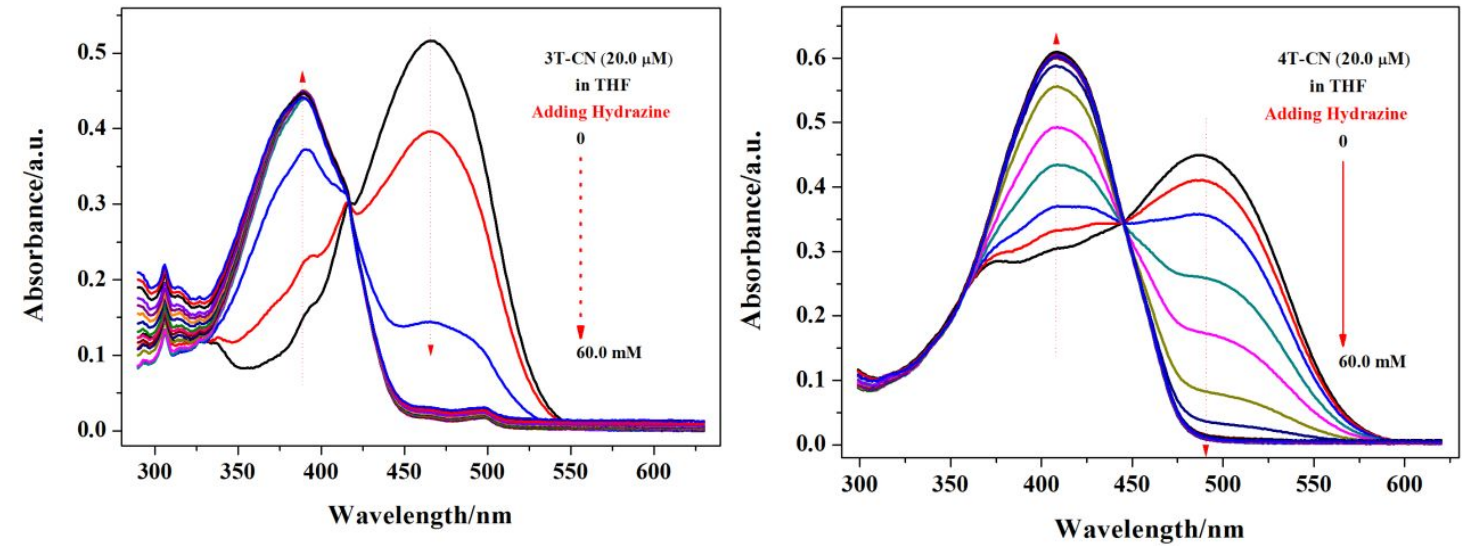

11. Optical response of the three spots-based sensor arrays to different assays and different concentrations of hydrazine

Figure S5. Optical response of the three spots-based sensor arrays using Zn-TPP, NA-3T-CN and their mixture as the probes under natural light and UV illumination.

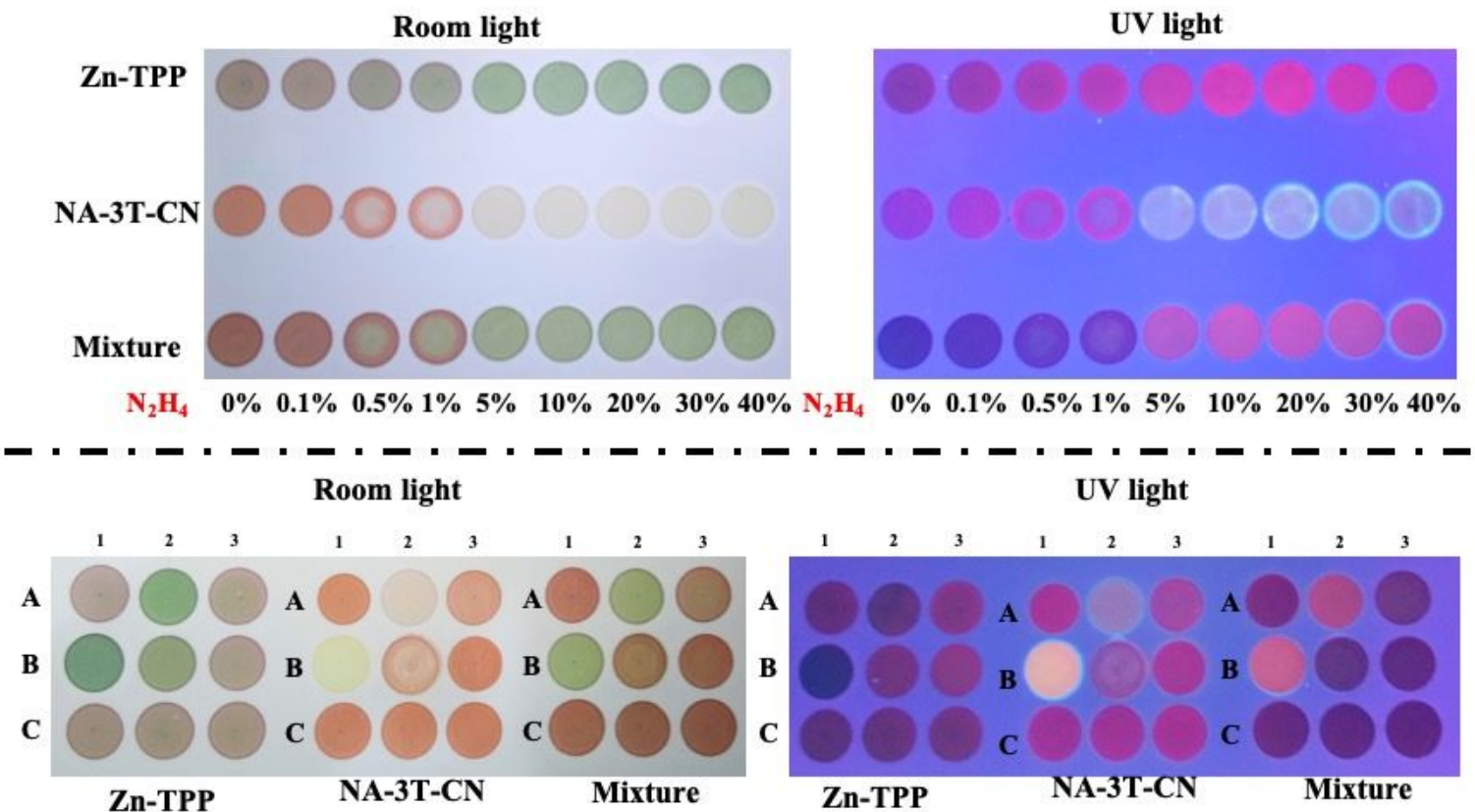

Notes : A1- $\mathrm{H}_{2} \mathrm{O} ; \mathbf{A 2}-\mathrm{N}_{2} \mathrm{H}_{4}$; A3-Methylamine; B1-Ethanolamine; B2-Aniline; B3-Diethylamine; C1-Diethanolamine; C2-Triethylamine; C3-Ammonium hydroxide. 


\section{NMR and HRMS spectra of the compounds}

Figure S6. ${ }^{1} \mathrm{H}$ NMR spectrum of compound $\mathbf{3 T - C N}$ in $d_{8}$-THF.

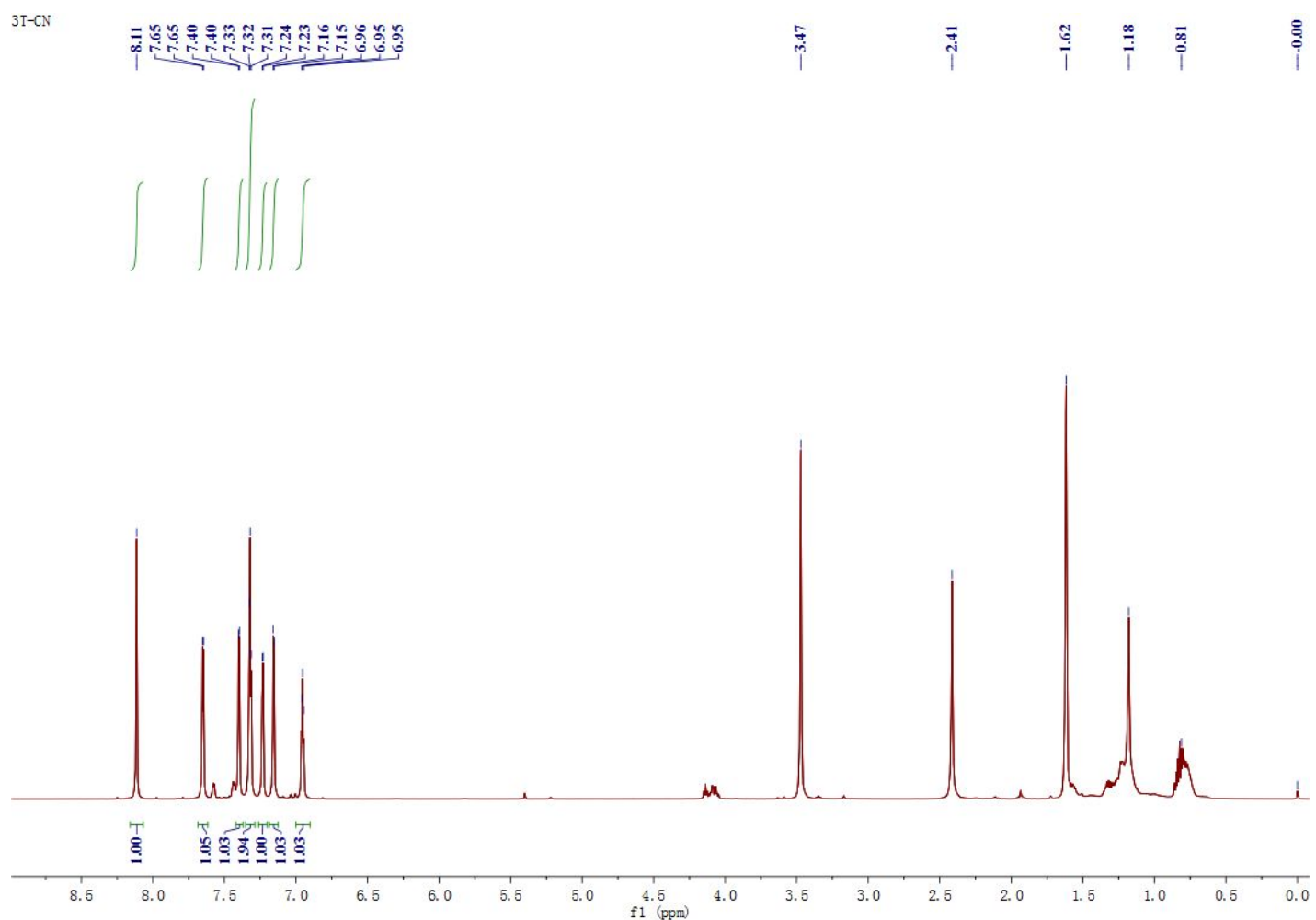

Figure S7. ${ }^{13} \mathrm{C}$ NMR spectrum of compound 3T-CN in $d_{8}$-THF.

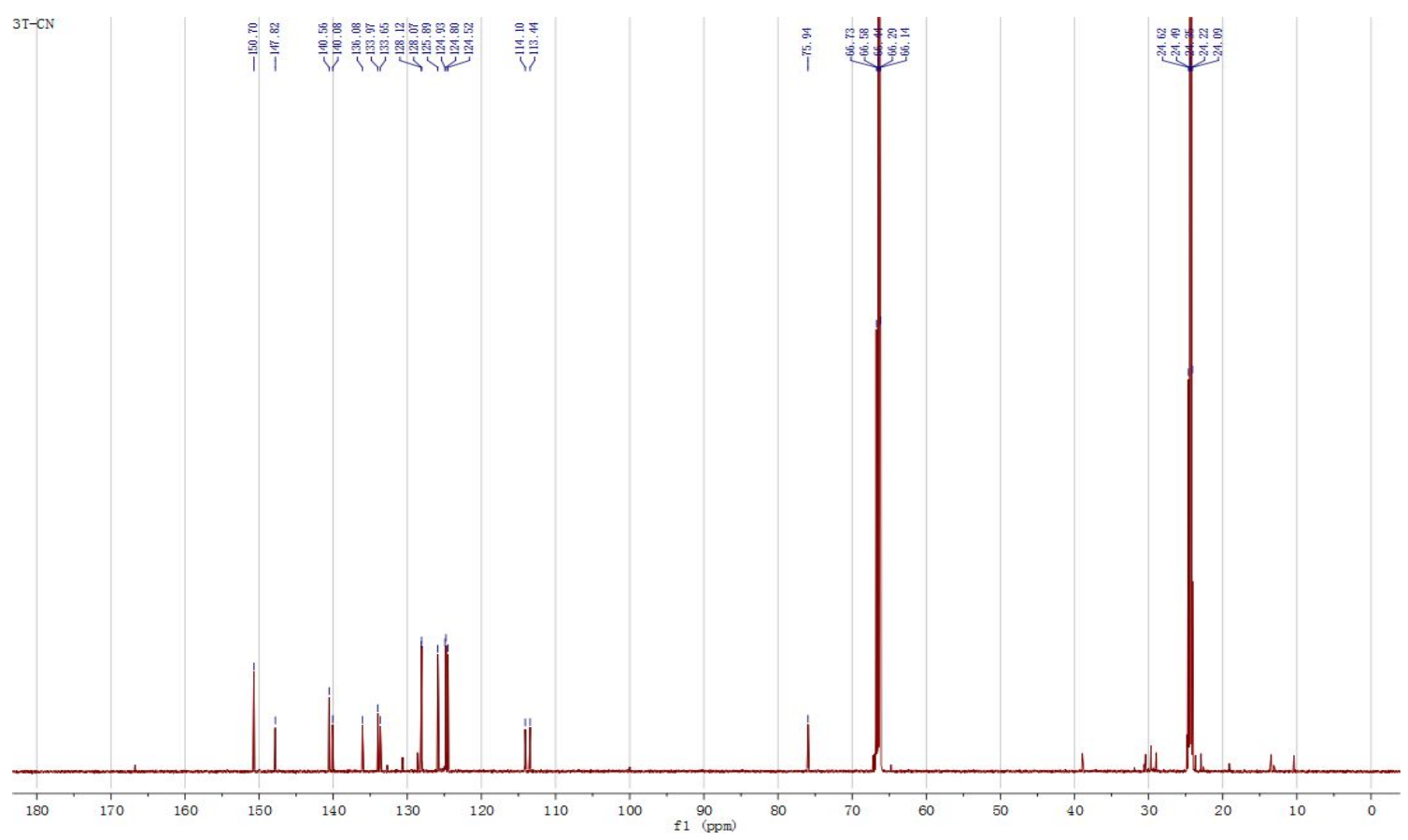


Figure S8. ${ }^{1} \mathrm{H}$ NMR spectrum of compound $4 \mathrm{~T}-\mathrm{CN}$ in $d_{8}$-THF.

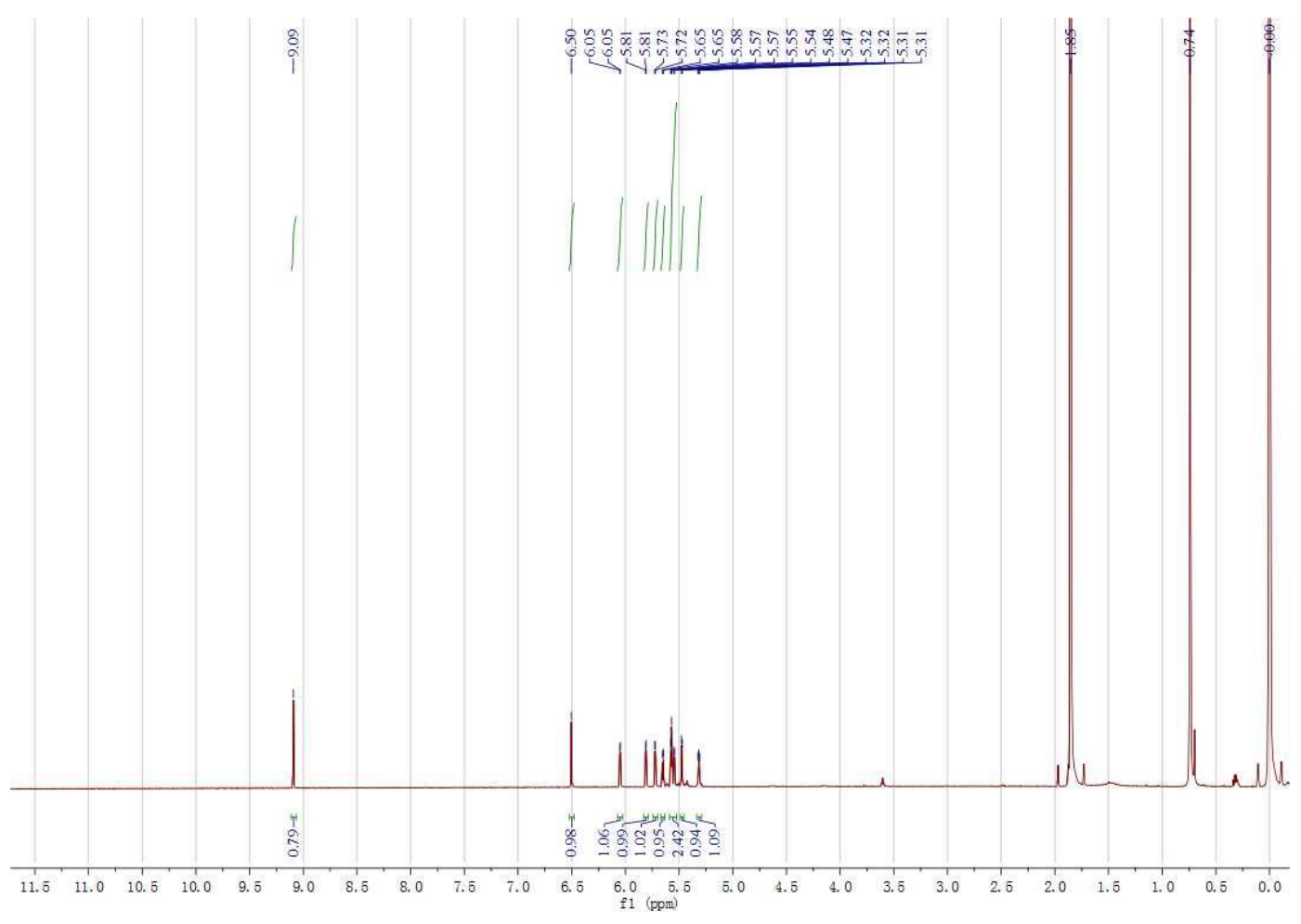

Figure S9. ${ }^{1} \mathrm{H}$ NMR spectrum of compound NA-3T-CN in $d_{8}$-THF.

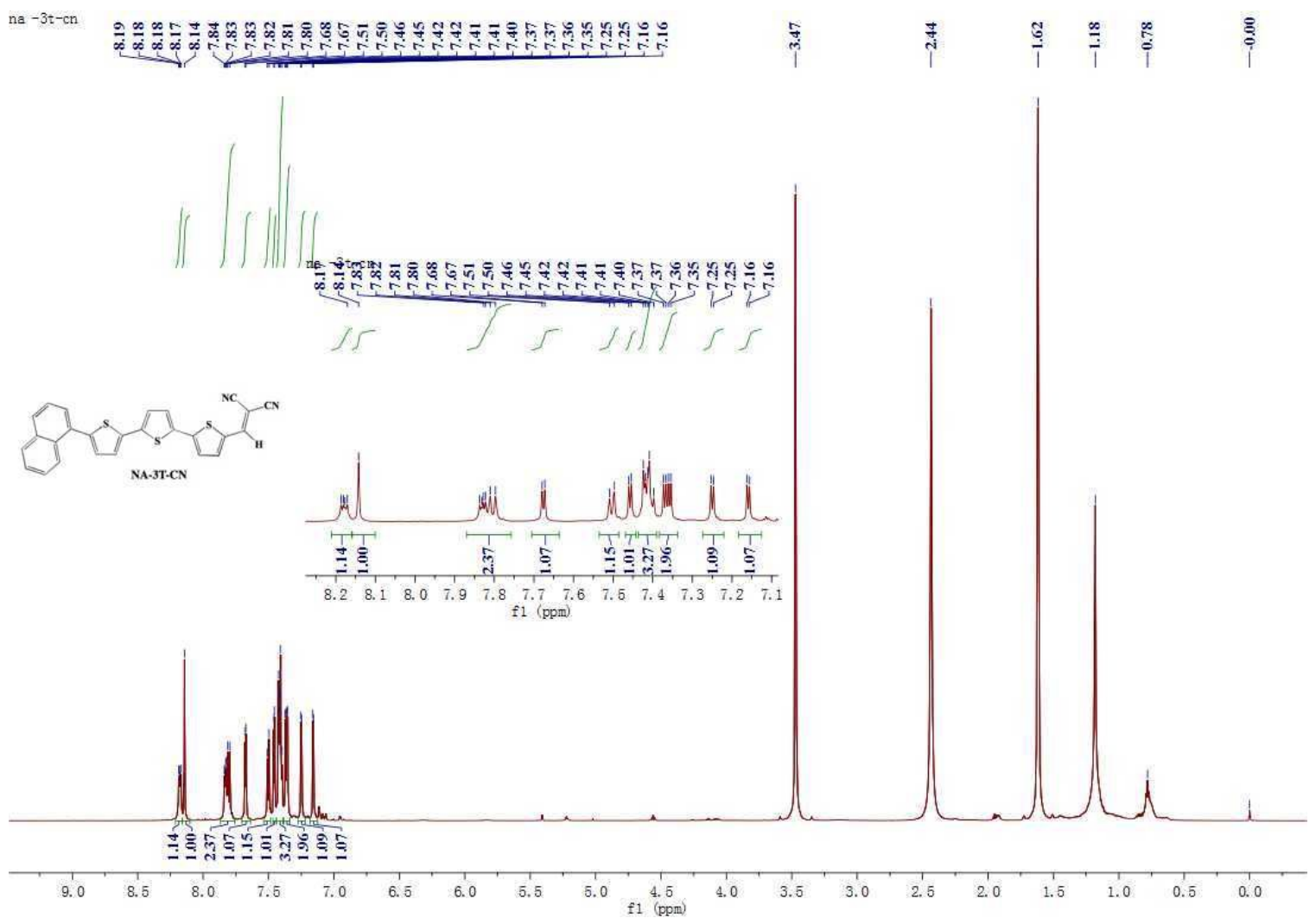


Figure S10. ${ }^{13} \mathrm{C}$ NMR spectrum of compound NA-3T-CN in $d_{8}$-THF.

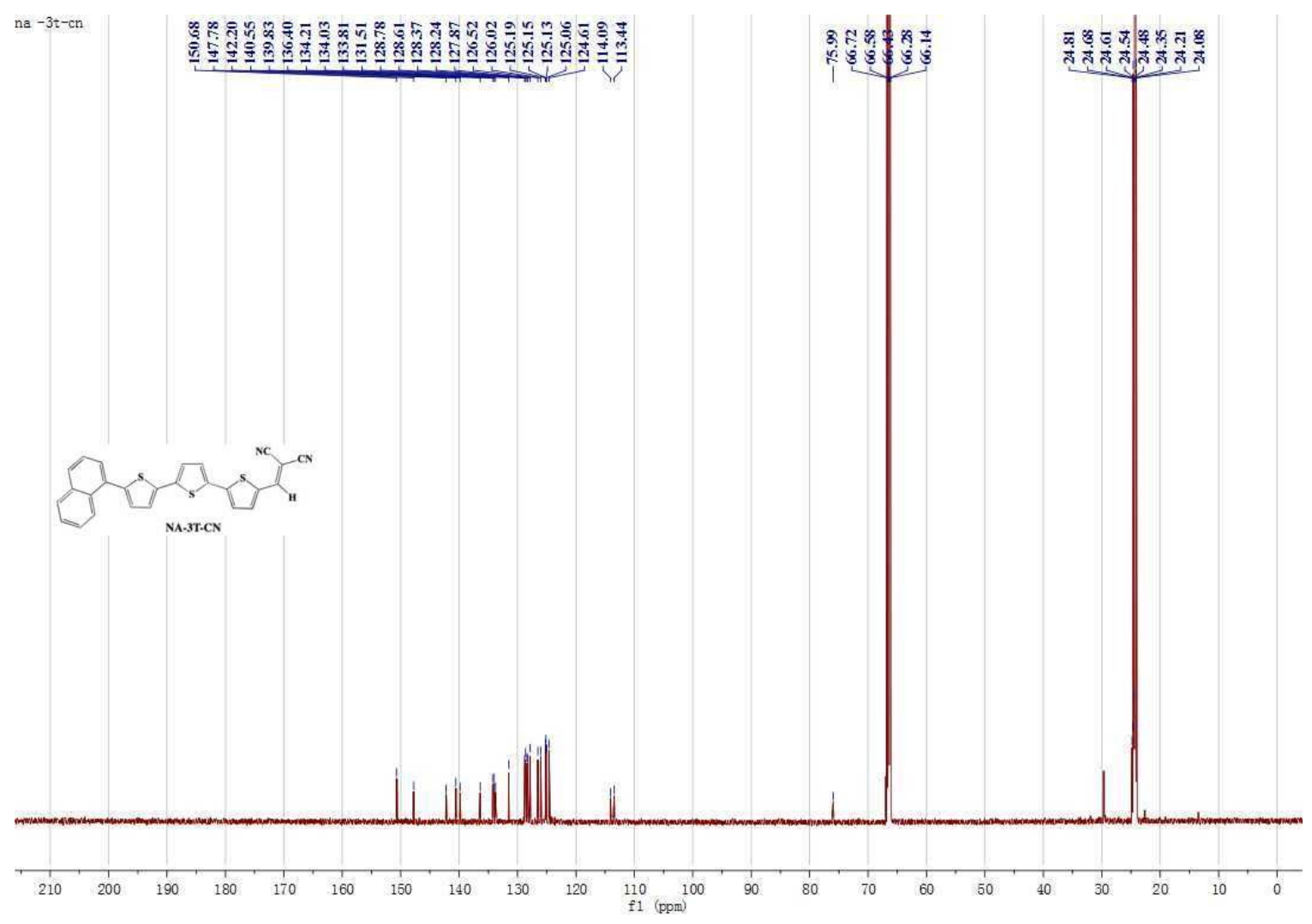

Figure S11. HR-MS spectrum of compound 3T-CN.

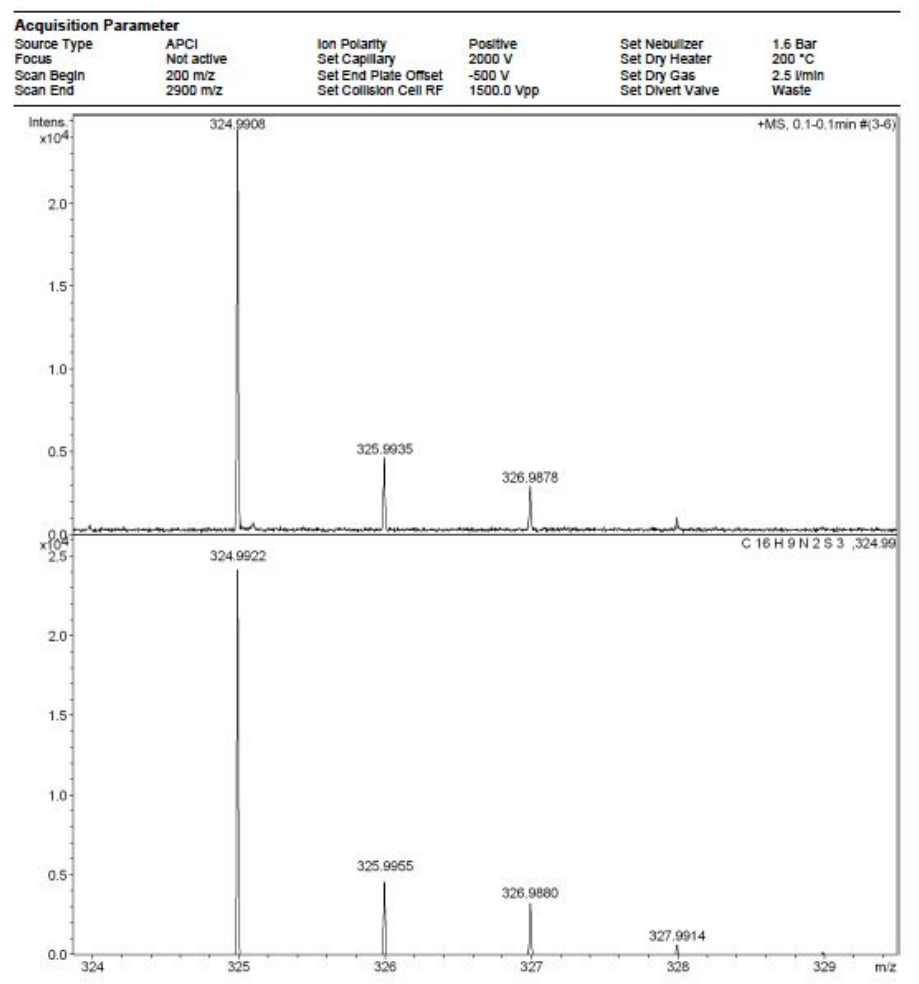


Figure S12. HR-MS spectrum of compound 4T-CN.

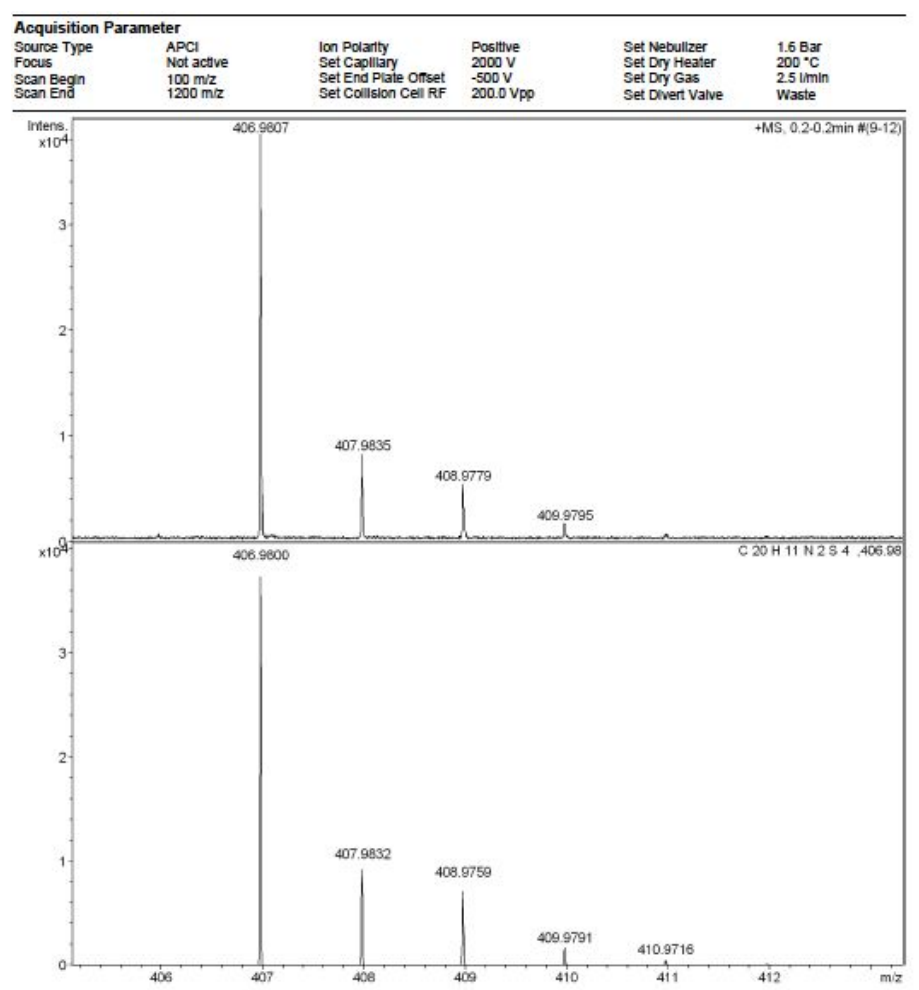

Figure S13. HR-MS spectrum of compound NA-3T-CN.

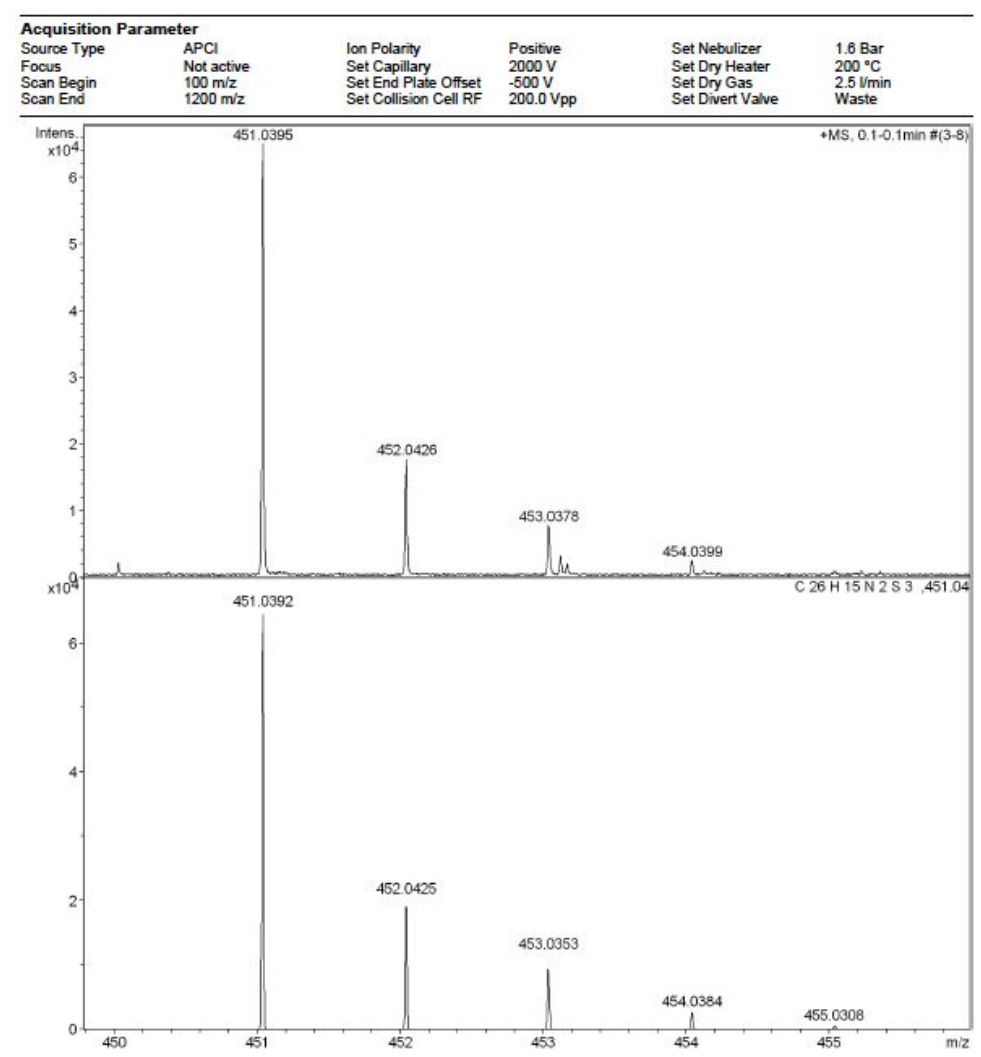


Figure S14. ${ }^{1} \mathrm{H}$ NMR spectrum of compound TPP.

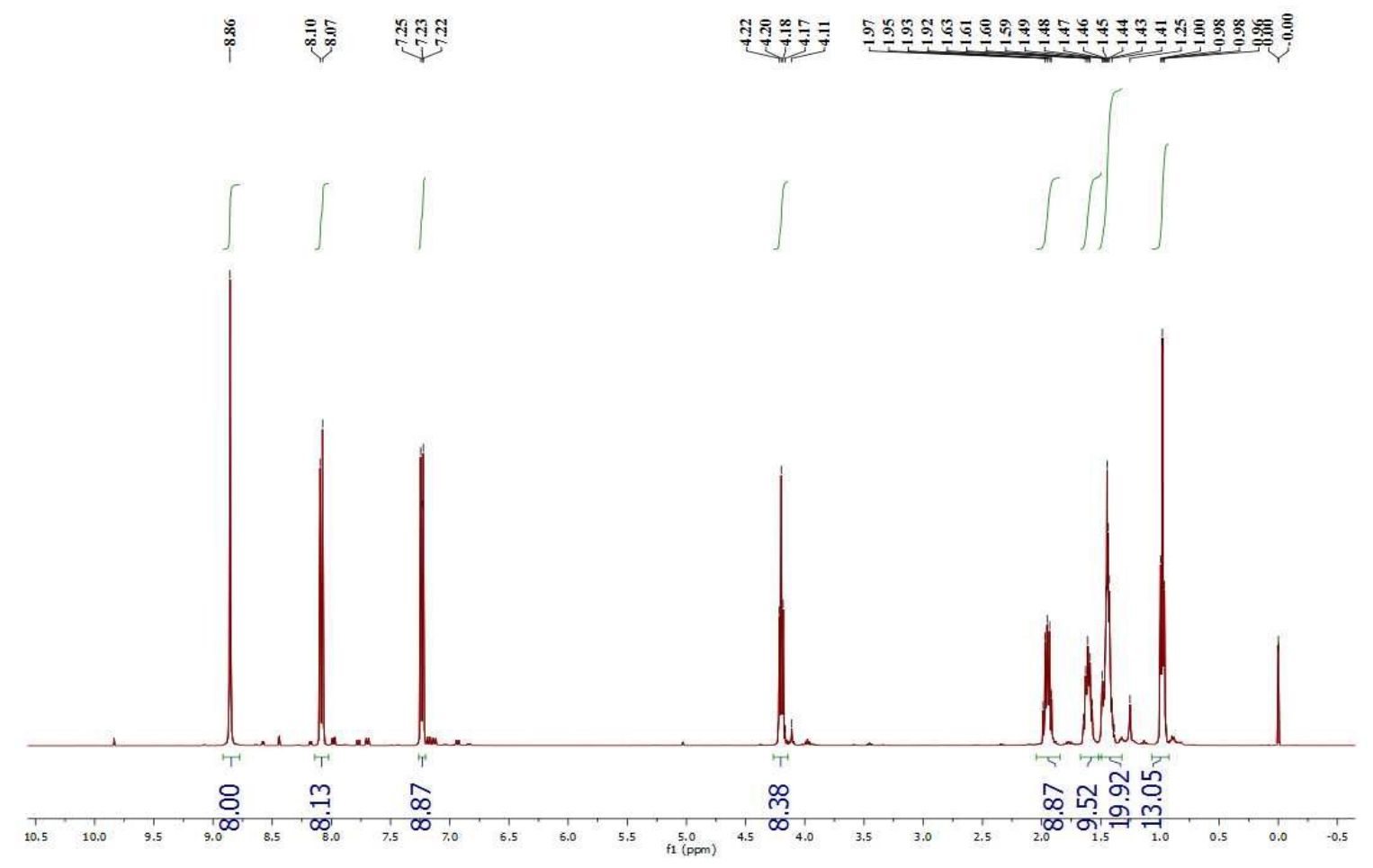

Figure S15. ${ }^{13} \mathrm{C}$ NMR spectrum of compound TPP.

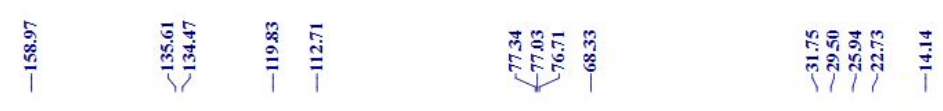

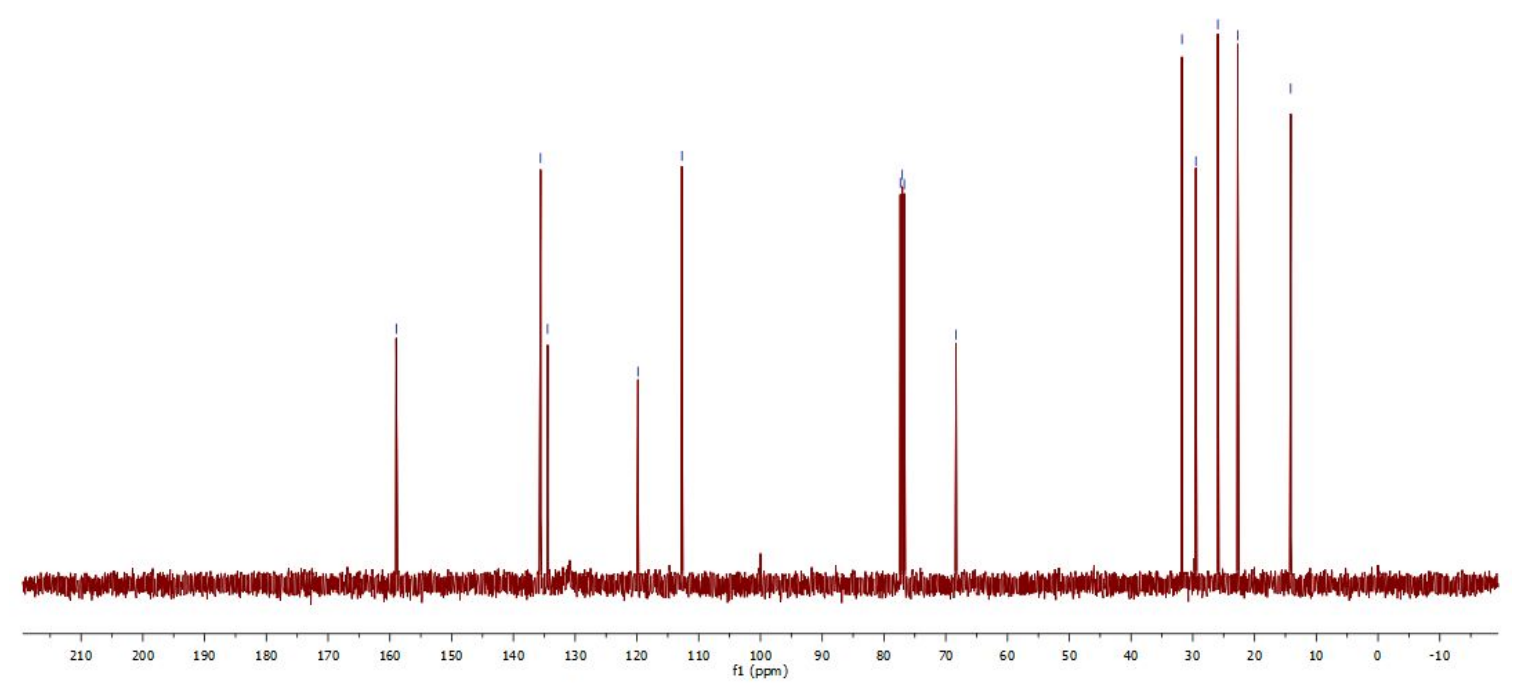


Figure S16. ${ }^{1} \mathrm{H}$ NMR spectrum of compound $\mathbf{Z n - T P P}$.

TPP-Zn

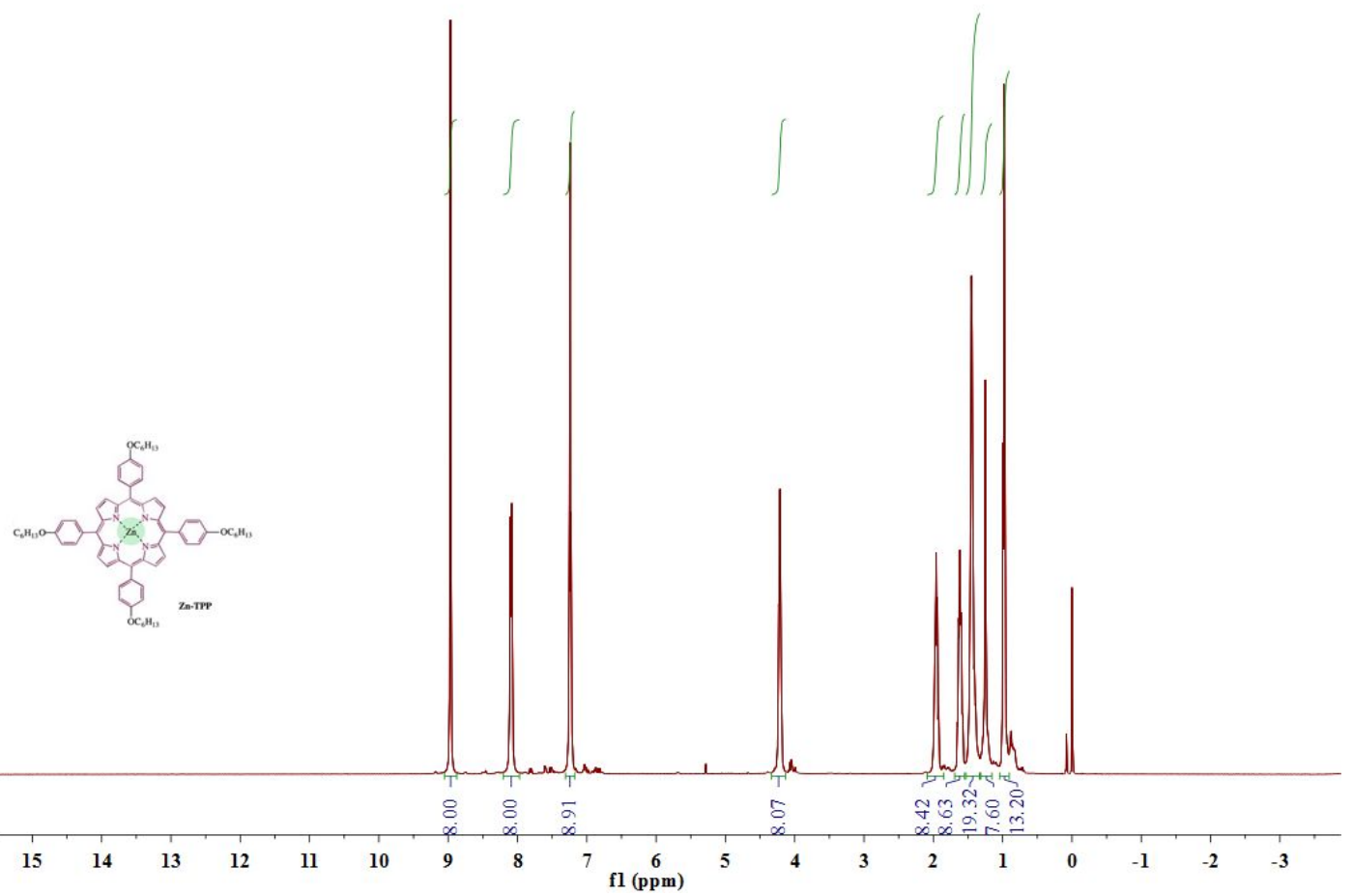

Figure S17. ${ }^{13} \mathrm{C}$ NMR spectrum of compound $\mathbf{Z n - T P P}$.

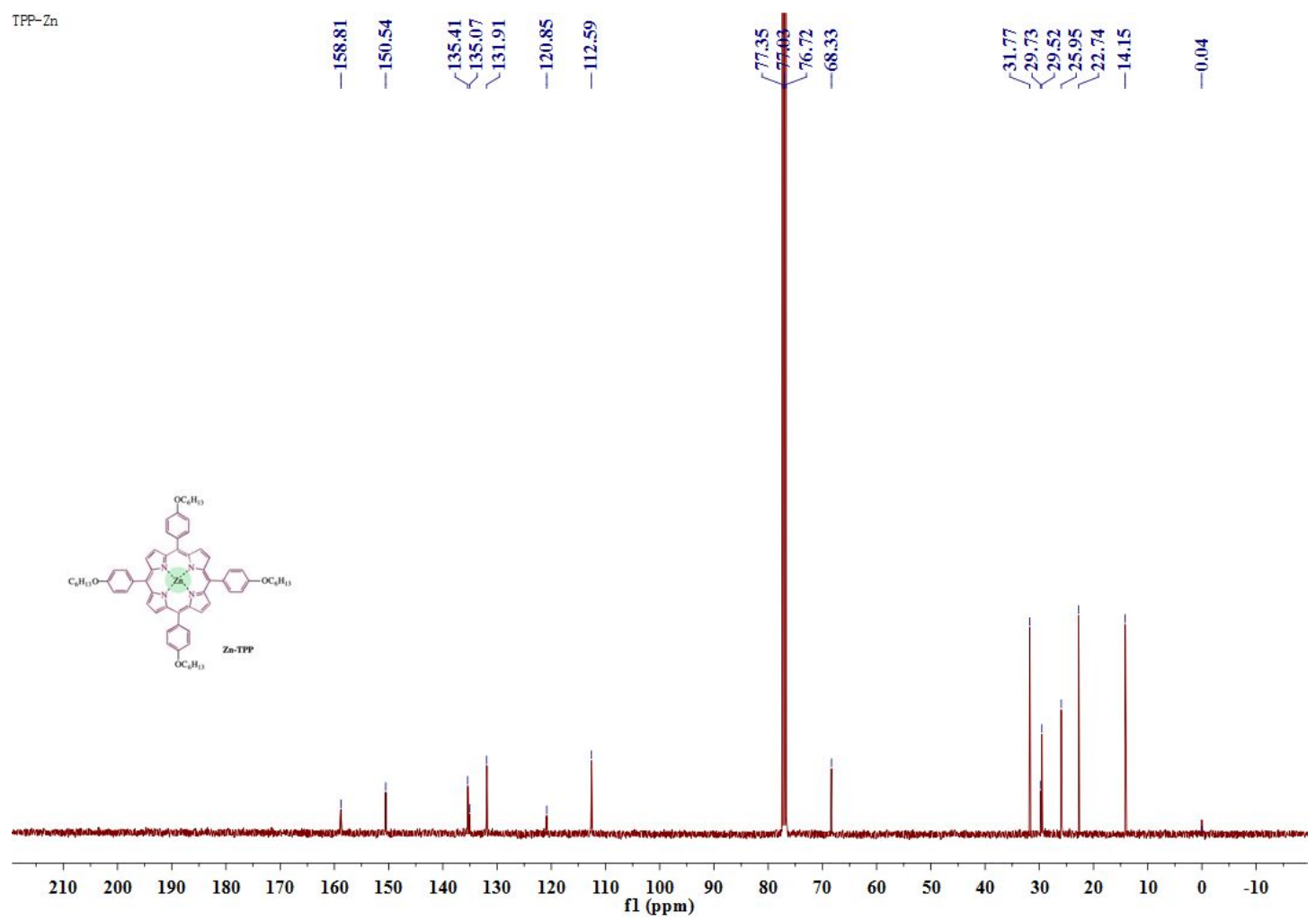


Figure S18. HR-MS spectrum of compound Zn-TPP.

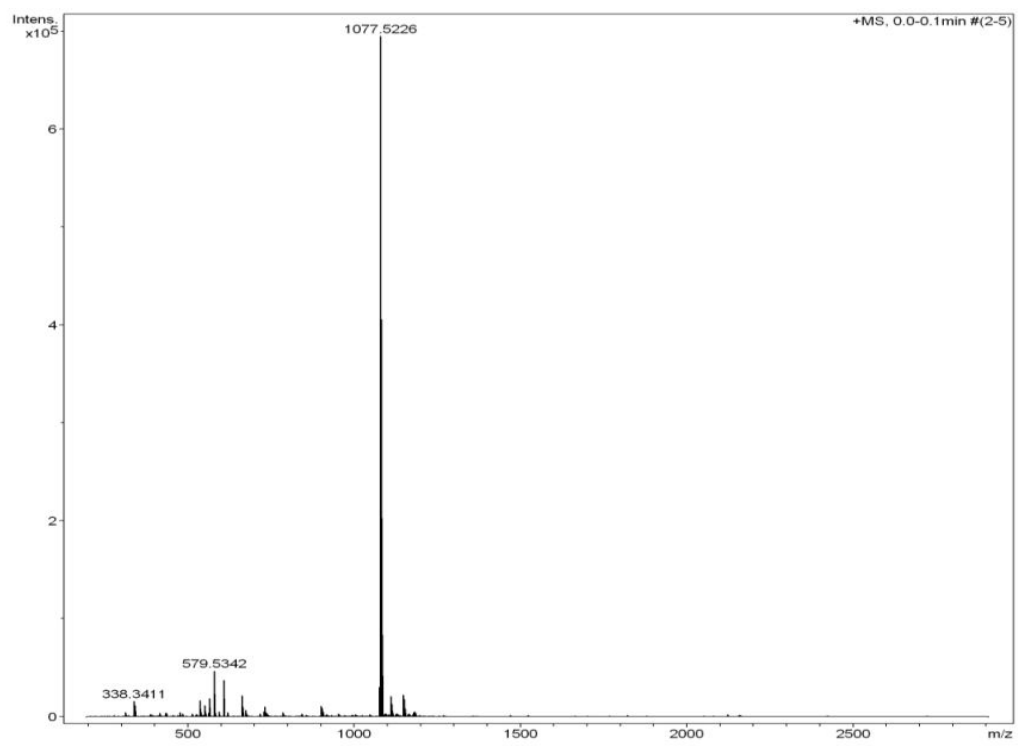

\section{Crystallographic data of NA-3T-CN}

Table S3. Crystal data and structure refinement for NA-3T-CN

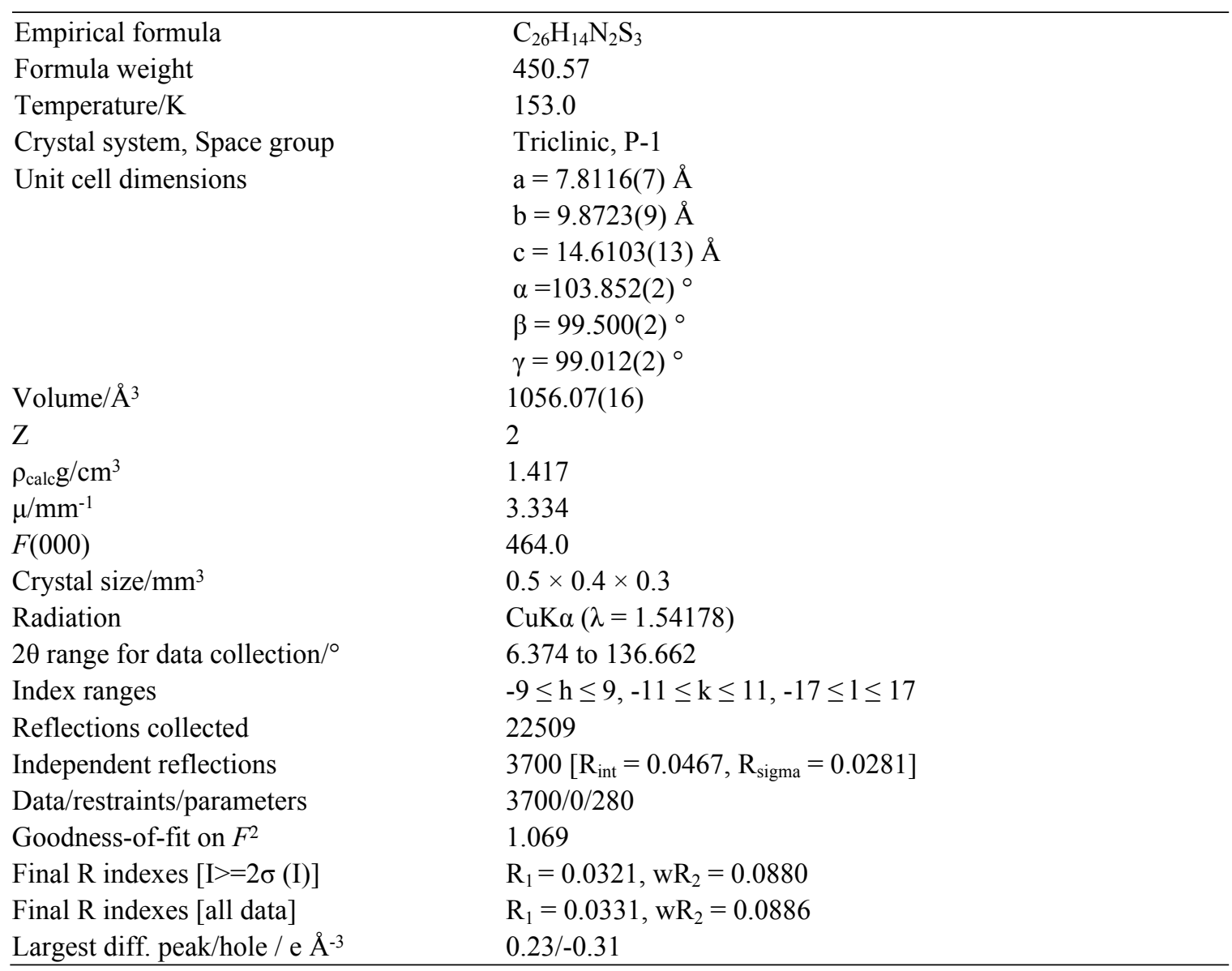




\section{A comparison of DL to other reported chemosensors for detecting hydrazine}

Table S4. A comparison of DL to other reported chemosensors for the $\mathrm{N}_{2} \mathrm{H}_{4}$ detection

\begin{tabular}{|c|c|c|c|c|}
\hline $\begin{array}{l}\text { Fluorescence } \\
\text { Chemosensor }\end{array}$ & $\begin{array}{c}\text { Media and } \\
\text { Response } \\
\text { Model }\end{array}$ & $\begin{array}{l}\text { Discrimination } \\
\text { Capability }\end{array}$ & DL/M & Reference \\
\hline 10 & $\begin{array}{l}\mathrm{THF} / \mathrm{H}_{2} \mathrm{O}(9 / 1 \\
v / v) ; \\
\text { fluorescence and } \\
\text { visual color } \\
\text { changes }\end{array}$ & $\begin{array}{l}\text { Hydrazine, ammonia, } \\
\text { dimethylamine, } \\
\text { triethylamine and } \\
\text { cyanide anion }\end{array}$ & $\begin{array}{c}5.82 \mathrm{mM} \\
\text { (Comp. 1), } \\
2.64 \mathrm{mM} \\
(\text { Comp. 2) } \\
\text { and } 1.28 \\
\mathrm{mM} \\
\text { (Comp. 3) }\end{array}$ & $\begin{array}{l}\text { Phys. Chem. } \\
\text { Chem. Phys., } \\
\text { 2019, 21, } \\
3218-3226\end{array}$ \\
\hline & $\begin{array}{l}\text { PBS buffer; } \\
\text { fluorescence and } \\
\text { visual color } \\
\text { changes }\end{array}$ & $\begin{array}{l}\text { High specificity for } \\
\text { hydrazine }\end{array}$ & $3.6 \times 10^{-8}$ & $\begin{array}{c}\text { J. Mater. } \\
\text { Chem. B, } \\
\text { 2018, } 6,8085- \\
8089\end{array}$ \\
\hline & $\begin{array}{l}\text { THF/Water }(2 / 8, \\
\text { v/v); } \\
\text { Fluorescence and } \\
\text { visual color } \\
\text { changes }\end{array}$ & Aliphatic amines & $3.67 \times 10^{-6}$ & $\begin{array}{c}\text { Analyst, 2018, } \\
\text { 143, 4298- } \\
4305\end{array}$ \\
\hline & $\begin{array}{l}\text { PBS buffer with } \\
2 \% \mathrm{CH}_{3} \mathrm{CN} ; \\
\text { Fluorescence and } \\
\text { visual color } \\
\text { changes }\end{array}$ & $\begin{array}{l}\text { High specificity for } \\
\text { hydrazine }\end{array}$ & $1.0 \times 10^{-7}$ & $\begin{array}{l}\text { Anal. Methods, } \\
\text { 2016, } 8,5832- \\
5837\end{array}$ \\
\hline & $\begin{array}{l}\text { Water/DMSO } \\
(4 / 6) ; \text { Off-On } \\
\text { fluorescence, }\end{array}$ & $\begin{array}{l}\text { High selectivity against } \\
\text { other amines }\end{array}$ & $0.3 \mathrm{ppb}$ & $\begin{array}{l}\text { Chem. } \\
\text { Commun. } \\
\text { 2014, } 50 \text {, } \\
1485-1487\end{array}$ \\
\hline & $\begin{array}{l}\text { Acetonitrile; } \\
\text { Turn-on } \\
\text { fluorescence }\end{array}$ & alkyl primary amines & No data & $\begin{array}{l}\text { J. Am. Chem. } \\
\text { Soc. 2014, } \\
\text { 136, 15493- } \\
15496\end{array}$ \\
\hline 3T-CN & $\begin{array}{l}\text { THF and TLC- } \\
\text { supported sensor } \\
\text { array; } \\
\text { Dual-model } \\
\text { (colorimetric and } \\
\text { fluorescence); } \\
\text { Visualization }\end{array}$ & $\begin{array}{l}\text { Among hydrazine, } \\
\text { aliphatic amines and } \\
\text { aromatic amine }\end{array}$ & $1.22 \times 10^{-5}$ & This Work \\
\hline
\end{tabular}




\section{References}

[1] H.-I. Je, J. Hong, H.-j. Kwon, N. Y. Kim, C. E. Park, S.-K. Kwon, T. K. An, Y.-H. Kim. Dyes Pigments 2018, 157: 93-100.

[2] M. M. Bader, P.-T. T. Pham, E. H. Elandaloussi. Cryst. Growth Des. 2010, 10: 5027-5030.

[3] Y. Xu, H. Li, X. Wu, Y. Chen, H. Hang, H. Tong, L. Wang. Polym. Chem. 2017, 8: 2484-2489.

[4] T. Liu, G. He, M. Yang, Y. Fang. J. Photochem. Photobiol. A: Chem. 2009, 202, 178-184.

[5] T. Liu, Y. Nie, G. He, Y. Zhang, L. Ding, Y. Fang. Chem. J. Chinese U. 2010, 31(3): 524-529.

[6] T. Liu, L. Ding, G. He, Y. Yang, W. Wang, Yu Fang. ACS Appl. Mater. Interfaces 2011, 3: 1245-1253.

[7] M. S. Wrackmeyer, M. Hummert, H. Hartmann, M. K. Riede, K. Leo. Tetrahedron 2010, 66: 87298733.

[8] A. Yassar, C. Videlot, A. Jaafari. Sol. Energy Mater. Sol. Cells 2006, 90: 916-922. 